admissions where diagnosed HIV prevalence in the local population exceeds two in 1000 population as well as for all patients presenting with certain indicator diseases. The aim of this audit was to determine if HIV testing rates of patients admitted with pneumonia improved after the implementation of opt-out testing for all acute medical admissions.

Methods HIV testing rates were compared for patients admitted with pneumonia before (September 2011) and after (September 2012) implementing opt-out testing for acute medical admissions. Patients were identified from hospital coding data for pneumonia during their inpatient stay. Electronic patient records were used to determine which patients had received a test for HIV during their admission

Results Seventy-nine patients were admitted with pneumonia in September 2011 and 86 in September 2012. Before opt-out HIV testing, 4/79 (5.1\%) patients were tested for HIV during their admission (mean age 63.5 years), with no positive tests. Following the implementation of opt-out testing, 22/86 (25\%) patients admitted with pneumonia were tested for HIV (mean age 62.5 years), with no patients testing positive. Since implementing opt-out HIV testing for acute medical admissions the rate of HIV testing in patients admitted with pneumonia increased from $5.1 \%$ to $25 \%(p=0.0002)$. Conclusion Following the implementation of opt-out HIV testing for acute medical admissions, the rate of testing in patients with a diagnosis of pneumonia has significantly increased. However, despite national guidelines and regional opt-out testing for acute medical admissions, a test was only performed in a quarter of eligible patients. Further work needs to be done in all areas of the hospital to increase awareness of HIV testing and to ensure rates of testing continue to rise.

\section{P2.043 ALBUMIN MAY INFLUENCE ELISA TEST RESULTS FOR HIV ANTIBODIES}

doi:10.1136/sextrans-2013-051184.0308

D Gupta, E Ganguly, S Das. Peerless Hospital and B.K Roy Research Centre, Kolkata, India

There may be interference of albumin in binding of HIV antibodies on HIV specific antigens. This experiment has been done to find out any such possible influence of albumin which may alter the serological test results.

Blood samples of known HIV positive patients were collected after taking consent. Total serum proteins were estimated, HIV antibody tests were performed with the collected samples directly and after mixing egg albumin to raise $25 \%$ of the baseline protein in each sample. The ELISA test for HIV antibodies in serums was performed with both types of samples and absorbance values were recorded.

It was found that after addition of egg albumin, the absorbance values were decreased in $66.0 \%$ samples and among them in $40.0 \%$ samples there was remarkable fall of absorbance levels. In the remaining $34.0 \%$ samples there was no change in absorbance values.

This study indicates that albumin present in the blood may influence outcome of ELISA test for HIV antibodies.

\section{P2.044 ONE BODY, ONE TEST, TWO LIVES: PATIENT CENTRED STRATEGY TO INCREASE HIV TESTING IN PREGNANT WOMEN AND THEIR PARTNERS}

doi:10.1136/sextrans-2013-051184.0309

L Kudryashova Hernandez. Neighborhood Health Services Corporation, Plainfield, NJ, United States

Background Neighborhood Health Service Corporation (NHSC), an urban community-based not-for-profit ambulatory health centre located in Plainfield, New Jersey, USA, provides prenatal services, labour and delivery to 750 uninsured, impoverished, minority women annually. Given that New Jersey has the third highest number of HIV women in the USA and the highest number of HIV positive children, early HIV detection and intervention in pregnant women become paramount. NHSC historically struggled with suboptimal HIV testing rates $(60 \%)$ in the pregnant patient population and needed to make radical programme changes to comply with CDC recommendations to ensure HIV testing is offered to $100 \%$ pregnant patients

Methods A PDSA (Plan-Do-Study-Act) cycle was conducted to test a new, patient-centred HIV testing approach: HIV Counselors are located in $\mathrm{OB}$ department; HIV counselling and on-the-spot Rapid testing are done at the time of initial $\mathrm{OB}$ registration; daily registration schedules are available to HIV Counselors at the beginning of workday; HIV results become part of $\mathrm{OB}$ records immediately upon result availability; educational DVDs are utilised in patient areas to increase awareness/interest among pregnant patients and their partners.

Results Per the revised and PDSA-improved strategy, NHSC achieved and sustained a 100\% compliance with CDC OB HIV testing recommendations over the past four years. Rapid HIV testing and Rapid-on-Rapid positive result confirmation allow for smooth and timely transition from HIV testing to the on-site HIV Early Intervention Services (EIS) for newly diagnosed HIV pregnant patients and their partners.

Conclusions The collected and analysed data suggests that convenient, coordinated, patient-centred approach to HIV counselling and testing among pregnant patients helps to: identify HIV positive pregnant patients early-on, preferably in the first/second trimesters immediately connect them to prenatal and HIV care and treatment services to minimise vertical HIV transmission; provide prevention services, HIV testing and treatment options for partners including prevention for positives.

\section{P2.045 SERIAL TESTING WITH AN INTERFERON-GAMMA RELEASE ASSAY IN HIV-1-INFECTED INDIVIDUALS}

doi:10.1136/sextrans-2013-051184.0310

'M C Aichelburg, ${ }^{2} \mathrm{~T}$ Reiberger, ${ }^{1} \mathrm{~F}$ Breitenecker, ${ }^{3} \mathrm{~A}$ Makristathis, ${ }^{1} \mathrm{~A}$ Rieger. 'Department of Dermatology, Division of Immunology, Allergy and Infectious Diseases (DIAID), Medical University of Vienna, Vienna, Austria; '2Department of Internal Medicine III, Division of Gastroenterology \& Hepatology, Vienna HIV \& Liver Study Group, Medical University of Vienna, Vienna, Austria; ${ }^{3}$ Department of Hygiene and Medical Microbiology, Division of Clinical Microbiology, Medical University of Vienna, Vienna, Austria

Background The clinical utility of serial screening for tuberculosis (TB) by interferon-gamma release assays has not been established in HIV-1-infected individuals.

Methods In this prospective study HIV-1-infected subjects underwent repeated QuantiFERON-TB Gold In-Tube assay (OFT-GIT) testing at baseline and after 24 months to determine the rate of conversions and reversions in a low TB-incidence country. Data on demographics, history of tuberculosis and HIV-1 parameters were obtained and risk factors associated with conversion or reversion of OFT-GIT results were assessed in a multivariate regression model.

Results Of 846 HIV-1-infected subjects, 9\% (76/846) were OFTGIT positive, $85 \%(718 / 846)$ were OFT-GIT negative and $6 \%$ $(52 / 846)$ OFT-GIT indeterminate at baseline, respectively. Concordant baseline and follow-up results were observed in $86 \%(686 / 794)$ of subjects. The observed inter-test agreement was 0.887 (95\% CI: 0.847-0.899) while the inter-test agreement of serial OFT-GIT testing was moderate (Cohan $\kappa$-coefficient $=0.448$ ). OFT-GIT conversions occurred in $9 \%(63 / 718)$ of individuals while OFT-GIT reversions were seen in $33 \%$ (25/76). Independent predictors for OFT-GIT conversion were origin from high $\mathrm{TB}$ incidence country $(\mathrm{OR}, 1.93 ; \mathrm{P}=0.024)$ and intravenous drug abuse $(\mathrm{OR}, 2.43$; $\mathrm{P}=0.016$ ). Of the 10 active TB cases during follow-up 5 had concordant positive OFT-GIT results and 2 were OFT-GIT converters. 
Conclusions TB screening in HIV-1 patients by serial OFT-GIT assays may be of clinical value due to its excellent reproducibility. However, OFT-GIT conversions and reversions should be interpreted carefully as their clinical significance remains to be established.

\section{P2.046 EVALUATION OF MATRIX-ASSISTED LASER DESORPTION/ IONIZATION TIME-OF-FLIGHT MASS SPECTROMETRY (MALDI-TOF-MS) FOR SPECIES IDENTIFICATION WITHIN THE NEISSERIA GENUS - EFFECTIVE ALTERNATIVE TO CONVENTIONAL TECHNIQUES?}

doi:10.1136/sextrans-2013-051184.0311

'D Golparian, ${ }^{2 B}$ Dauphin, 'B Hellmark, ${ }^{3 B}$ Claesson, 'M Unemo. 'WHO Collaborating Centre for Gonorrhoea and other STIs, Department of Laboratory Medicine, Microbiology, Örebro University Hospital, ÖREBRO, Sweden; ${ }^{2}$ Assistance Publique-Hôpitaux de Paris, Paris, France; ${ }^{3}$ Department of Clinical Microbiology, Skaraborg Hospital, Capio Diagnostic AB, Skövde, Sweden

Background Highly specific and sensitive discrimination between closely related pathogenic and commensal Neisseria spp is crucial because these species frequently colonise the same anatomical sites. Herein, two commercially available Matrix-Assisted Laser Desorption/Ionization Time-of-Flight Mass Spectrometry (MALDI-TOFMS) platforms and one independent software and database (Andromas) was compared to conventional phenotypic and genetic tests routinely used for identification of Neisseria spp.

Methods The performance of each platform, analysing 129 pathogenic isolates (Neisseria gonorrhoeae and Neisseria meningitidis) and 69 commensal Neisseria isolates (15 different species), was determined by deposition of one single culture colony to the MALDI plate and analysed in Microflex (Bruker, Germany) and VITEK MS (bioMérieux, France) according to the manufacturer's instructions. Subsequently, the acquired data from Microflex was submitted for analysis in the Andromas software and database (Andromas, France), which uses a different algorithm for species identification. Unfortunately, VITEK MS data is not compatible with Andromas. Phenotypic and genetic (16S rRNA gene sequencing) methods were used for final discrepancy analysis (still pending).

Results Microflex correctly identified all (100\%) N. gonorrhoeae and $\mathrm{N}$. meningitidis, however, four commensal isolates were indicated as possible N. meningitidis. Three of these four isolates were $\mathrm{N}$. kochii. The VITEK MS misidentified $1 \mathrm{~N}$. gonorrhoeae, $1 \mathrm{~N}$. meningitidis and 2 commensal isolates (both $\mathrm{N}$. kochii) were indicated as possible N. meningitidis. Finally, Microflex data analysed in the Andromas software and database correctly identified all (100\%) pathogenic and commensal strains.

Conclusion This study shows that both Microflex and VITEK MS discriminate pathogenic Neisseria species from commensal Neisseria species with a high, but not ideal, specificity. Furthermore, an optimal MALDI-TOF-MS platform should be compatible with secondary softwares and databases for confirmation. Importantly, the Microflex results analysed in the secondary software and database Andromas correctly identified all (100\%) pathogenic and commensal strains.

\section{P2.047 EVALUATION OF A MULTIPLEX REAL-TIME PCR ASSAY FOR RAPID DETECTION OF C.TRACHOMATIS AND $N$. GONORRHOEAE FROM GENITAL CLINICAL SPECIMENS}

doi:10.1136/sextrans-2013-051184.0312

'M Screm, 'M Di Santolo, 'C Scarparo, ${ }^{1,2} \mathrm{~A}$ Arzese. 'Microbiologia, Azienda Ospedaliero-Universitaria, Udine, Italy; ${ }^{2}$ Department of Experimental Clinical Medicine, University of Udine, Udine, Italy

Background Sexually transmitted diseases (STDs) are among the most common causes of illness in the world, being associated to acute disease, infertility, long term disability and death. In particular the prevalence of C.trachomatis and $N$. gonorrhoeae infections appear to remain steady or increasing, although both diagnostic and therapeuthical tools are available, at least in developed countries. Standard diagnostic protocols can be complex, time-consuming, and sensitivity of culture methods affected by specimen characteristics. In addition, certified methods are today required, especially in the management of cases of sexual abuse.

Methods Multiplex real-time PCR method (Xpert CT/NG, Cepheid) has been tested versus $N$. gonorrhoeae standard culture and C. trachomatis molecular assay (Artus C. trachomatis TM PCR Kit, Qiagen). 45 clinical samples (female and male urogenital swabs, ocular infections, first void of urine, seminal fluid, and external quality control samples - UK NEOAS) were selected and stored at $20^{\circ} \mathrm{C}$. Xpert CT/NG allowed to perform in one-step extraction, amplification and detection of C.trachomatis and N. gonorrhoeae DNA directly from all the variety of selected clinical specimens, within $60 \mathrm{~min}$

Results $100 \%$ results agreement was found between Xpert CT/NG and standard protocols, including expected results by UK-NEOAS quality assessment specimens. However, batch PCR is a multi-step time-consuming process, the turn-around-time (TAT) required from 8 to $48 \mathrm{hrs}$ versus the single technical and computer-assisted interpretation step by Xpert CT/NG, resulting in a $<$ two hrs TAT.

Conclusions Xpert CT/NG test is a easy, rapid, accurate and certified method in routine diagnostics of $N$. gonorrhoeae and C. trachomatis infections.

\section{P2.048 EVALUATION OF TWO COMMERCIAL REAL-TIME PCR ASSAYS FOR DETECTION OF MYCOPLASMA GENITALIUM IN UROGENITAL SPECIMENS}

doi:10.1136/sextrans-2013-051184.0313

${ }^{1,2} \mathrm{C}$ Le Roy, ${ }^{1,2,3}$ S Pereyre, ${ }^{1,2,3}$ C Bébéar. 'University of Bordeaux, Bordeaux, France; 2Institut National de la Recherche Agronomique, Bordeaux, France, ${ }^{3}$ Centre Hospitalier Universitaire de Bordeaux, Bordeaux, France

Objectives Mycoplama genitalium is a sexually transmitted organism associated with non-gonococcal urethritis in men and several inflammatory reproductive tract syndromes in women. Nucleic acid amplification tests are currently the only available methods for detection. The first commercially available real-time (RT-) PCR kits have been recently developed. We compared the TIB MOLBIOL LightMix $^{\circledR}$ Kit Mycoplasma genitalium and the Diagenode Mycoplasma genitalium real-time PCR kit to the in-house TaqMan RTPCR used routinely for the M. genitalium diagnostic.

Methods DNA extracts from $50 \mathrm{M}$. genitalium-negative and $53 \mathrm{M}$ genitalium-positive urogenital specimens collected between January 2010 and May 2011 at the Bordeaux University hospital, France, were retrospectively and systematically selected and thawed. DNA had been extracted using the MagNA Pure DNA isolation kit I (Roche Diagnostics). DNA extracts were evaluated by the TIBMOLBIOL LightMix ${ }^{\circledR}$ Kit Mycoplasma genitalium and the Diagenode Mycoplasma genitalium real-time PCR kit (DIA-MG-050 vs2) in comparison with a M. genitalium in-house RT-PCR targeting the $\mathrm{MgPa}$ adhesin gene using the cobas z480 analyser (Roche Diagnostics)

Results The in-house PCR was first evaluated using two thermal cyclers, LC480 and cobas z480 (Roche Diagnostics). As no significant difference was noted, the cobas z480 was used in the rest of the study. The clinical sensitivity was $98 \%, 92 \%$ and $100 \%$ for the LightMix ${ }^{\circledR}$ Kit Mycoplasma genitalium, the Diagenode Mycoplasma genitalium real-time PCR kit and the in house RT-PCR, respectively. The clinical specificity was 100\% for both kits and $94 \%$ for the in house RT-PCR. There was no statistically significant difference between the clinical sensitivity and specificity of these 3 methods. Moreover, there was no statistically significant difference between 